\title{
Implementation of Tube Radial Distribution Chromatography by Using a Commercially Available HPLC System
}

\author{
Hyo Kan,* Kento Yamada,* Nobuyuki Sanada,* Koyo NAKata,* and Kazuhiko Tsukagoshi*,**† \\ *Department of Chemical Engineering and Materials Science, Faculty of Science and Engineering, \\ Doshisha University, Kyotanabe, Kyoto 610-0321, Japan \\ **Bio-Microfluidic Science Research Center, Kyotanabe, Kyoto 610-0321, Japan
}

\begin{abstract}
Tube radial distribution chromatography based on tube radial distribution flow, or annular flow, in an open-tubular capillary has been reported. The chromatographic system requires specific instruments and treatments for microfluidic flow in the capillary tube. In this study, we have developed a new model of tube radial distribution chromatography, which is comprised of a commercially available HPLC system without any packed separation columns. Separation is performed in an open-tubular pipe $\left(100-\mu \mathrm{m}\right.$ inner diameter and $350-\mathrm{cm}$ length; temperature, $\left.5^{\circ} \mathrm{C}\right)$ connected between the pump and the detector in the HPLC system. An analyte solution is introduced with a sample injector $(2-\mu \mathrm{L}$ volume $)$ and a ternary water/acetonitrile/ethyl acetate mixed solution (volume ratio of 3:8:2) is delivered as an eluent solution into the pipe at a flow rate of $10-\mu \mathrm{L} \mathrm{min}{ }^{-1}$. Fused silica and stainless pipes can separate 1-naphthol and 2,6-naphthalenedisulfonic acid, but a polyetheretherketone pipe cannot. The obtained data provides an important clue to practical developments in separation science.
\end{abstract}

Keywords Phase-separation multiphase flow, tube radial distribution chromatography, open-tubular pipe, fused-silica, stainless

(Received September 15, 2017; Accepted September 25, 2017; Published February 10, 2018)

\section{Introduction}

Conventional multiphase flow, which is called immiscible multiphase flow, is generated by mixing water and a hydrophobic organic solvent solution in a tube to provide a kinetic liquidliquid interface in the flow. ${ }^{1-3}$ We have studied a new type of multiphase flow in a microspace, called "phase-separation multiphase flow", which contrasts with the immiscible multiphase flow. ${ }^{4}$ Two-phase separation mixed solutions, such as a ternary water/hydrophilic/hydrophobic organic mixed solution, are separated through a phase transformation by changing the temperature and/or pressure into upper and lower phases in a batch vessel. When homogeneous two-phase separation mixed solutions are delivered into a microspace and the temperature and/or pressure are changed, a phase transformation occurs, leading to a phase separation multiphase flow.

We are especially interested in stable annular flow possessing inner and outer phases provided by the phase-separation multiphase flow. ${ }^{5}$ Such specific microfluidic behavior and flow are called the "tube radial distribution phenomenon" (TRDP) and the "tube radial distribution flow" (TRDF), respectively., We have developed capillary chromatography based on TRDF, where the inner and outer phases in the annular flow work as mobile and pseudo-stationary phases in chromatography, respectively. We refer to this new type of chromatography as

$\dagger$ To whom correspondence should be addressed.

E-mail: ktsukago@mail.doshisha.ac.jp "tube radial distribution chromatography" (TRDC). ${ }^{4-6}$

TRDC requires specific instruments and treatments for microfluidic flow, such as gravity sample injection (several tens $\mathrm{nL})$, a microflow solution delivery $\left(<1 \mu \mathrm{L} \mathrm{min}^{-1}\right)$, tube length $(<100 \mathrm{~cm})$, and on-capillary detection. In this study, we propose the first model of TRDC using a commercially available HPLC system comprising a pump, an injector, and a detector. These conditions (sample injection volume, flow rate, and pipe length) are quite different from the previous ones in TRDC.

\section{Experimental}

Water was purified using an Elix 3 UV (Millipore, Billerica, MA). All reagents were commercially available and of analytical grade. 1-Naphthol, 2,6-naphthalenedisulfonic acid (2,6-NDS), Eosin Y, perylene, acetonitrile, and ethyl acetate were purchased from Wako Pure Chemical Industries (Osaka, Japan). Open-tubular pipes, such as fused-silica, stainless-steel (SUS316L), and polyetheretherketone (PEEK), with an inner diameter of $100 \mu \mathrm{m}$, were purchased from GL Science (Tokyo, Japan).

Figure 1 shows a schematic diagram of the present TRDC comprised of a commercially available HPLC system (solution delivery pump, LC-20AT, Shimadzu; UV-VIS detector, SPD20A, Shimadzu; and sample injector $(2 \mu \mathrm{L})$, Type-7725, Rheodyne). An open-tubular pipe (350 cm in length) was connected between the pump and the detector in the HPLC system. The pipe temperature was controlled by submerging the pipe into a beaker of water maintained at a fixed temperature 
$\left(5^{\circ} \mathrm{C}\right)$ while stirring. The ternary mixed solution of water/ acetonitrile/ethyl acetate (volume ratio of 3:8:4) was delivered as an eluent solution at a flow rate of $10 \mu \mathrm{L} \mathrm{min}$. The model analyte solution $(2 \mu \mathrm{L})$ was injected and separated through the pipe, and then detected with the spectrophotometric detector (at $254 \mathrm{~nm}$ ).

We set up a pipe made of fused-silica with a size equivalent to that used in the TRDC system on a fluorescence microscope. The pipe temperature was controlled with a thermo plate. The fluorescence in the pipe was monitored at approximately $300 \mathrm{~cm}$ from the pipe inlet using the fluorescence microscope (BX51; Olympus, Tokyo, Japan) equipped with a $\mathrm{Hg}$ lamp, optical filters (U-MWU2; 330 - 385-nm excitation filter and >420 nm emission filter), and a CCD camera (JK-TU53H; Toshiba, Tokyo, Japan). The eluent solution contained $0.1 \mathrm{mM}$ perylene and $1 \mathrm{mM}$ Eosin Y. We delivered the eluent solution into the pipe at a flow rate of $10 \mu \mathrm{L} \mathrm{min}{ }^{-1}$ using the delivery pump.

Commercially available HPLC system

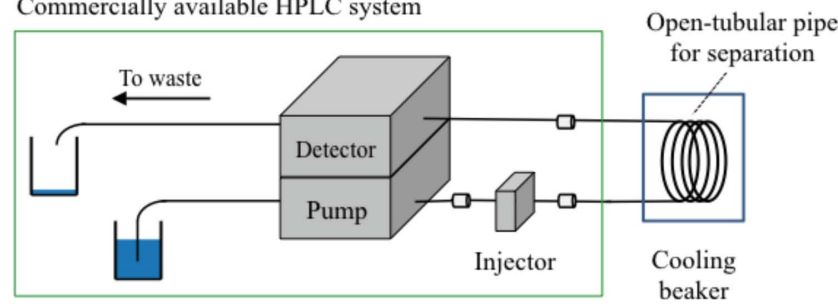

Fig. 1 Schematic diagram of the present TRDC comprised of a commercially available HPLC system. Open-tubular pipe for separation $(100-\mu \mathrm{m}$ i.d. and 350-cm length) fused-silica, stainlesssteel, or PEEK pipe (300-cm cooling length); eluent solution, water/ acetonitrile/ethyl acetate mixed solution (volume ratio, 3:8:4); flow

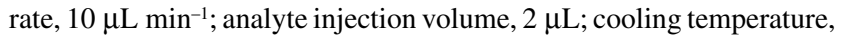
$5^{\circ} \mathrm{C}$; and detection wavelength, $254 \mathrm{~nm}$.

\section{Results and Discussion}

Figure 2 shows a phase diagram of the water/acetonitrile/ethyl acetate mixed solution, including the solubility curves between homogeneous and heterogeneous solutions at 20 and $5^{\circ} \mathrm{C}$. A composition of the ternary water/acetonitrile/ethyl acetate homogeneous solution (volume ratio of $3: 8: 4$ ) as an eluent solution is positioned near the solubility curve at $20^{\circ} \mathrm{C}$ on the phase diagram. The homogenous solution (single phase) at $20^{\circ} \mathrm{C}$ changes to a heterogeneous solution (two phases) at $5^{\circ} \mathrm{C}$ through a phase transformation in the batch vessel.

On the other hand, when the ternary mixed solution is delivered into a pipe made of fused-silica, changing the temperature (from room temperature to $5^{\circ} \mathrm{C}$ ) at a flow rate of $10 \mu \mathrm{L} \mathrm{min}{ }^{-1}$ allows TRDF in the pipe to be observed using a fluorescence microscope-CCD camera system. The obtained fluorescence photographs are shown in Fig. 2. TRDF is not naturally observed in the pipe at $20^{\circ} \mathrm{C}$, but is clearly observed at $5^{\circ} \mathrm{C}$, where the organic solvent-rich major inner phase (perylene, blue) and the water-rich minor outer phase (Eosin Y, green) are generated. These results confirm that the phase-separation multiphase flow or TRDF occurs in the open-tubular fusedsilica pipe equipped with a commercially available HPLC system under the present conditions. An observation of such fluidic behavior in a stainless-steel or PEEK pipe could not be performed due to the opacity of the pipe material.

A model mixed analyte solution (1-naphtol and 2,6-NDS, $1.0 \mathrm{mM}$ each) was subjected to the present TRDC, where the open-tubular pipe composed of fused-silica, stainless-steel, or PEEK was connected as a separation pipe to a commercially available HPLC system. Stainless-steel and PEEK pipes are normally used as connecting pipes in a HPLC system. 1-Naphthol and 2,6-NDS are not separated in the system at $20^{\circ} \mathrm{C}$ for all of the pipes. However, they are separated and detected at $5^{\circ} \mathrm{C}$ in chromatogram for the fused-silica and

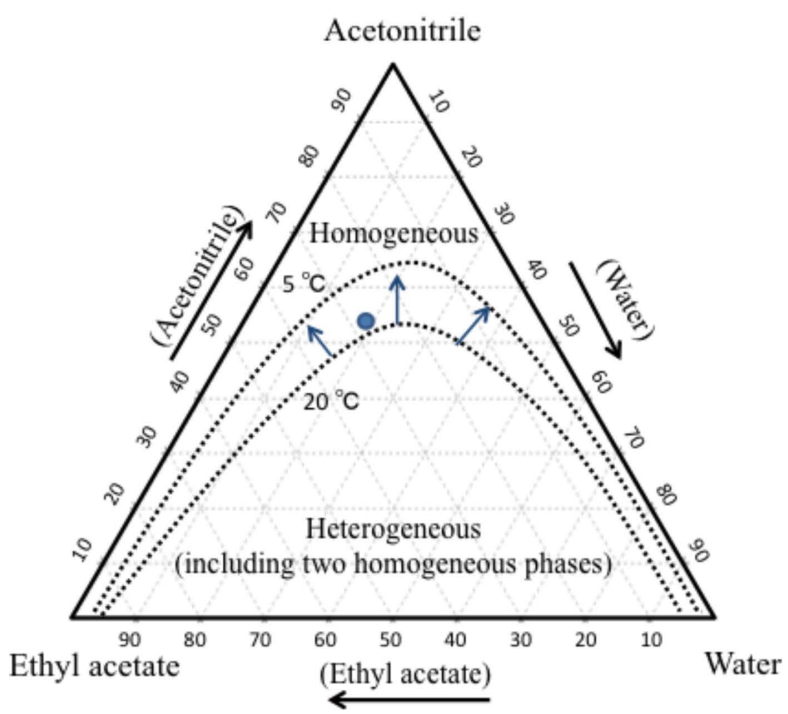

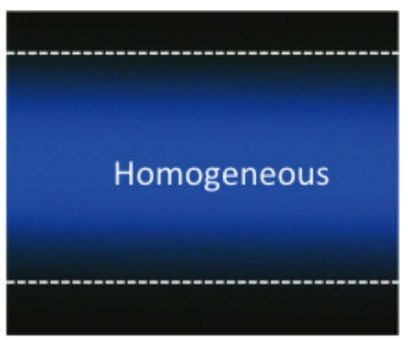

$20^{\circ} \mathrm{C}$

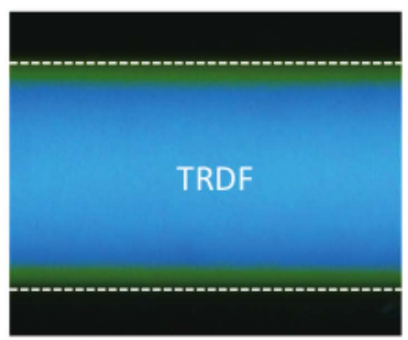

$5{ }^{\circ} \mathrm{C}$

Fig. 2 Phase diagram of the ternary water/acetonitrile/ethyl acetate mixed solution including solubility curves at 20 and $5^{\circ} \mathrm{C}^{7}$ as well as fluorescence photographs of the ternary mixed solution in the separation pipe composed of fused-silica. Photographs are for the water/acetonitrile/ethyl acetate mixed solution (volume ratio of $3: 8: 4$ ) at a flow rate of $10 \mu \mathrm{L} \mathrm{min}{ }^{-1}$ with temperatures of 20 and $5^{\circ} \mathrm{C}$. 
(a)

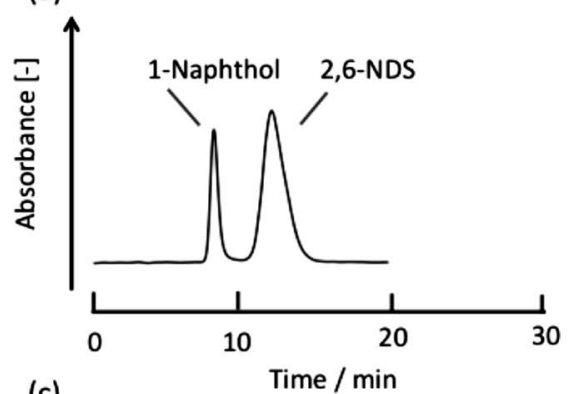

(c)

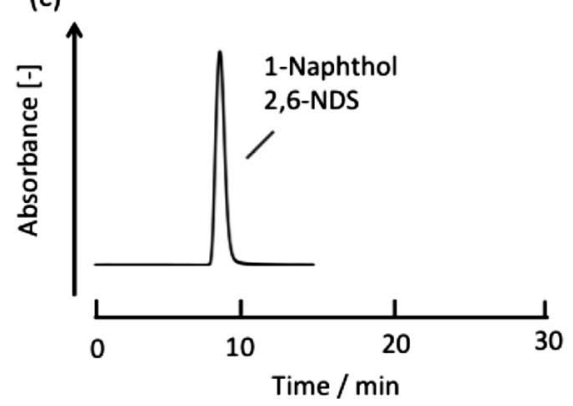

(b)

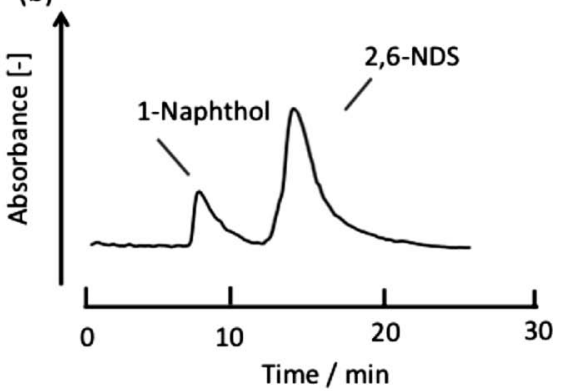

Fig. 3 Chromatograms obtained with the present TRDC comprised of a commercially available HPLC system. (a) Fused-silica, (b) stainless-steel, and (c) PEEK pipe. Analytical conditions are described in Fig. 1.

stainless-steel pipes, but not for the PEEK pipe (Fig. 3).

With the organic solvent-rich eluent solution (ternary water/ acetonitrile/ethyl acetate mixed solution, 3:8:4 volume ratio), the organic solvent-rich major inner phase and the water-rich minor outer phase are generated. The outer phase works as a pseudo-stationary phase under laminar flow conditions. The analytes are distributed between the inner (mobile) and the outer (pseudo-stationary) phase based on their partitioning ability, undergoing chromatographic separation.

1-Naphtol and 2,6-NDS are separated in the fused silica and stainless-steel pipes, through a chromatographic process based on TRDC, although formation of TRDF in the stainless-steel pipe is not confirmed by the fluorescence microscope-CCD camera system. The reason that the HPLC system equipped with the PEEK pipe does not separate the model mixture remains unclear. First of all, we examined the contact angles of the ternary water/acetonitrile/ethyl acetate mixed solution (volume ratio of 3:8:4) to each plate of glass, stainless, and PEEK. The contact angles to glass, stainless, and PEEK are $30.3,22.3$, and 39.2 degree, respectively. The large contact angle of the solution to PEEK means that the relatively low affinity between them may be related to non-TRDF or nonseparation.

\section{Conclusions}

We successfully developed TRDC comprised of a commercially available HPLC system with a pump, an injector, and a detector. Separation is performed in pipes composed of either fused-silica or stainless, which are connected between the pump and the detector in the HPLC system. The analytical conditions between the present system and the previous one in TRDC are quite different. Although we must examine the analytical conditions in the HPLC system in detail, the obtained data provides an important clue for outstanding practical developments of capillary HPLC.

\section{Acknowledgements}

This work was supported by a Grant-in-Aid for Scientific Research (B) from the Ministry of Education, Culture, Sports, Science and Technology of Japan (No. 17H03083).

\section{References}

1. M. Kashid and L. K. Minsker, Chem. Eng. Process., 2011, 50, 972.

2. H. Foroughi and M. Kawaji, Int. J. Multiphase Flow, 2011, $37,1147$.

3. J. Jovanovic, E. V. Rebrov, T. A. X. Nijhuis, M. T. Kretzer, V. Hessel, and J. C. Schouten, Ind. Eng. Chem. Res., 2012, 51,1015 .

4. K. Tsukagoshi, J. Flow Injection Anal., 2015, 32, 89, and references cited therein.

5. K. Tsukagoshi, Anal. Sci., 2014, 30, 65, and references cited therein.

6. S. Fujinaga, M. Hashimoto, K. Tsukagoshi, and J. Mizushima, Anal. Sci., 2016, 32, 455.

7. Y. Tanigawa, S. Fujinaga, M. Hashimoto, and K. Tsukagoshi, Anal. Sci., 2012, 28, 921. 